\title{
Predicting species-specific responses of fungi to climatic variation using historical records
}

\author{
JEFFREY M. DIEZ*, TIMOTHY Y. JAMES†, MARSHALL MCMUNN $\ddagger$ and INÉS IBÁÑEZ§ \\ *Institute of Integrative Biology, ETH, Zürich 8007, Switzerland, †Department of Ecology and Evolutionary Biology, University \\ of Michigan, Ann Arbor, MI 48104, USA, \$Department of Entomology, University of California, Davis, CA 95616, USA, §School \\ of Natural Resources and the Environment, University of Michigan, Ann Arbor, MI 48104, USA
}

\begin{abstract}
Although striking changes have been documented in plant and animal phenology over the past century, less is known about how the fungal kingdom's phenology has been changing. A few recent studies have documented changes in fungal fruiting in Europe in the last few decades, but the geographic and taxonomic extent of these changes, the mechanisms behind these changes, and their relationships to climate are not well understood. Here, we analyzed herbarium data of 274 species of fungi from Michigan to test the hypotheses that fruiting times of fungi depend on annual climate and that responses depend on taxonomic and functional groups. We show that the fungal community overall fruits later in warmer and drier years, which has led to a shift toward later fruiting dates for autumn-fruiting species, consistent with existing evidence. However, we also show that these effects are highly variable among species and are partly explained by basic life-history characteristics. Resulting differences in climate sensitivities are expected to affect community structure as climate changes. This study provides a unique picture of the climate dependence of fungal phenology in North America and an approach for quantifying how individual species and broader fungal communities will respond to ongoing climate change.
\end{abstract}

Keywords: climate change, fungi, mycorrhizal, phenology, precipitation, saprotrophic, temperature

Received 16 March 2013 and accepted 26 April 2013

\section{Introduction}

The timing of phenological events has wide-ranging ecological and evolutionary consequences via effects on species' demography, species interactions (Hegland et al., 2009; Encinas-Viso et al., 2012), and ecosystem functions such as carbon sequestration (Richardson et al., 2010). Phenology has also been an early indicator of changing climate, as a wide variety of phenological events have been shown to be changing over time and in response to climatic trends (Root et al., 2005; Menzel et al., 2006). However, most of these changes in phenology have been documented in plants and animals, and the phenology of the fungal kingdom has only recently begun to be evaluated (Gange et al., 2007; Büntgen et al., 2012, 2013). Given the important roles fungi have in the carbon cycle (Orwin et al., 2011; Clemmensen et al., 2013), as a food resource in forest ecosystems (Fogel \& Trappe, 1978), and through their many associations with plants as pathogens, mutualists, and decomposers, their response to climate needs to be better understood if we want to forecast future ecosystem changes.

Recent studies in Europe have shown that the timing of fruiting (mushroom production) in some fungal communities has changed in the last half century.

Correspondence: Jeffrey M. Diez, tel. +41 44632 9883, fax +41 44 632 1271, e-mail: jeffrey.diez@env.ethz.ch
Fall-fruiting fungi have shown average delays in fruiting between 1.2 and 3.4 days per decade in Norway, United Kingdom, Austria, and Switzerland (Kauserud et al., 2008, 2012; Büntgen et al., 2012), and spring fungi have been fruiting 3.8 days earlier per decade on average in Norway and United Kingdom (Kauserud et al., 2010b). In addition to these changes in mean trends over time, there appears to be considerable variation among species and among regions (Gange et al., 2007; Pinna et al., 2010; Kauserud et al., 2012), and the fruiting season also appears to be widening in these four European countries (Kauserud et al., 2012). However, historical trends over time cannot accurately foretell future changes under climate change because the past and predicted rate of climate change is not constant. Also, a variety of factors in addition to climate, such as forest age, nitrogen deposition, land use, and $\mathrm{CO}_{2}$ concentrations, are also changing over time and may influence phenology (Egli, 2011). Therefore, to understand future phenological responses to climate change, relationships between phenology and climate need to be quantified directly. Previous studies have reported suggestive correlations between fungal phenology and temperature and rainfall (Straatsma et al., 2001; Gange et al., 2007; Kauserud et al., 2010b; Büntgen et al., 2012), but the interactions between temperature and precipitation have not been quantified. 
Also unlike plants, the expectations for how fungal phenology should respond to climate are not well established. Warmer and wetter conditions generally enhance the growth of fungal mycelium in culture, but the relationship between mycelial growth and fruiting in nature is uncertain and a variety of nutritional, environmental, and biotic factors can influence fruiting (Kües \& Liu, 2000; Moore et al., 2008). Nonetheless, a couple of general hypotheses are plausible for explaining how climate can affect the timing of fungal fruiting. If fruiting is dependent on the accumulation of sufficient energy over a growing season (Krebs et al., 2008), then species would be expected to fruit earlier in warmer and wetter years. Alternatively, if fruiting is triggered by climatic cues signaling the end of suitable climatic conditions for mycelial growth, fruiting should be later in warmer years. However, the combination of direct and indirect effects of climate on fungi further complicates these predictions. Fungi may respond directly to the temperature and moisture of the soil or substrate (e.g., leaf litter or wood), but also indirectly via plant-mediated resource dynamics (i.e., carbon from mycorrhizal root associations or litter production for saprotrophic species). These indirect relationships suggest different predictions for saprotrophic and mycorrhizal species (Gange et al., 2007). One prediction is that mycorrhizal fungal species' responses should directly follow host plant phenology because they rely on carbon fixed each year. Saprotrophic species, on the other hand, use carbon created in previous years, suggesting that their phenology may reflect direct rather than resource-mediated relationships with climate. Fruiting of saprotrophic species in laboratory settings is sometimes initiated when resources are depleted or barriers to growth are reached (Moore et al., 2008), although it remains unclear whether this translates to field settings. If fruiting is a response to depleted resources, then warmer and wetter conditions should lead to earlier fruiting as species deplete their resources more quickly. Conversely, mycorrhizal species may continue to draw resources from plant hosts, which themselves have an extended growing season (Piao et al., 2007), and delay fruiting to reflect the extended growing season. These hypotheses suggest that responses of macrofungal fruiting may appear idiosyncratic, yet dependent on the source of carbon that the fungal species is utilizing.

Here, we present results from the first long-term, multispecies study of fungal phenology in North America. We use a dataset of fungal fruiting times from Michigan to test the overall hypothesis that climate determines fruiting dates of fungi. Specifically, we use over 13000 records ranging from 1904 to 2004 from the University of Michigan fungal herbarium for 274 species of fungi from 64 genera to ask the following questions: first, how has the timing of fruiting responded to climatic variation during the last century?; second, have these climate sensitivities led to changes in fungal fruiting dates over time?; and finally, we tested the hypotheses that species differ in their responses to climate and trends over time, and that lifehistory characteristics help to explain differences among species. We test all hypotheses using a unique application of multilevel regression models that account for the large within-species variation while testing hypotheses at the species level.

\section{Materials and methods}

\section{Species data}

The University of Michigan herbarium has over 180000 mushroom specimens, with over 1/3 from Michigan, representing over 17000 species and 2500 genera. Over 60000 fungal specimens have been data based and were available for this study. Mushroom collections can be used to examine fungal phenology because the short life span of many species' fruiting bodies mean that collection dates are reasonable estimates of fruiting dates (Kauserud et al., 2008). Despite the large quantity, these data present several challenges, common to herbarium data, for understanding patterns of fruiting. To minimize potential biases, we established several criteria for inclusion of records in the compiled database. First, records must have complete information for date (day/month/year) and county/state of collection. We determined county to be the lowest common spatial resolution of records, and therefore matched climate with records at the county level (described below). Second, we removed records that were duplicates of the same species from the same day and same county. Third, we limited analyses to species that are well collected and have suitable temporal and spatial coverage. We only analyzed species with at least 20 records from the state, at least 10 records associated with one climate station, and with collections spanning at least 3 decades. Finally, we removed plant pathogens and taxa with persistent fruiting bodies from the dataset to be analyzed. This selection process left us with 12578 records from 274 species and 64 genera, distributed across 59 counties. Using available textbooks and online resources, these species were classified as either mycorrhizal, saprotrophic, or unknown trophic behavior, and as associated with deciduous trees, evergreen trees, both, or unknown. A table of species included in the study, along with traits and estimated climate relationships, is given in Table S1.

\section{Climate data}

Climate data were obtained from the US Historical Climatology Network (USHCN), consisting of daily and monthly climate data from roughly 1200 climate stations distributed across continental United States. We matched each county in which fungal collections were made to the nearest climate 
station. The areas encompassed by the nine climate stations used in Michigan are referred to below as climate zones. The climate variables used for predicting each species' phenology were the mean monthly temperature and the mean monthly precipitation during the 3 months prior to each species' mean fruiting date. Three months was chosen as a reasonable period of time that reflects the biological important period of time leading up to fruiting for each species.

\section{Statistical modeling}

Responses to climate. The uneven geographic distribution of herbarium data complicates efforts to estimate relationships between fruiting date and time. Because fruiting date changes across latitudes due to broad climatic gradients, the geographic location of collections needed to be accounted for in all analyses. We accounted for these geographical differences in fruiting dates by using multilevel regression models with separate intercept terms for each climate zone (area covered by each climate station, described above). Other studies have accounted for geographic variation by using fruiting dates normalized by a spline regression on latitude and longitude (Kauserud et al., 2012). We prefer the multilevel modeling framework because it not only accounts for broad latitudinal trends in fruiting dates but also accounts for differences in fruiting dates due to other unknown conditions that vary spatially but may affect phenology, such as forest age and composition, nitrogen deposition, and land-use patterns.

In the first model, we assumed fixed climate relationships across all species, but allowed regression intercepts to vary by climate zone. The dates of fruiting were modeled as a function of temperature and precipitation as follows:

$$
\begin{aligned}
\text { FruitDate }_{s, y, z}= & \alpha_{s, z}+\beta \cdot \text { temp } * \text { Temp }_{y, z}+\beta \cdot \text { precip } * \text { Precip }_{y, z} \\
& +\beta \cdot \text { int } * \text { Precip }_{y, z} * \text { Temp }_{y, z}+\epsilon_{s, y, z}
\end{aligned}
$$

where FruitDate $s, y, z$ is the raw data of collection dates for species $s$, in year $y$, and climate zone $z$.

The intercept, $\alpha$, was allowed to vary for each species and climate zone combination. These intercepts were modeled hierarchically with each zone level intercept being drawn from an overall intercept for the species, such that $\alpha_{s, z} \sim$ Normal $\left(\alpha . s p_{s}, \sigma_{\mathrm{s}}^{2}\right)$. This hierarchical structure helps prevent outlier values in zones with more limited data by allowing estimates in each county to be informed by data in other counties through the overall species-level distribution. The regression coefficients, $\beta$.temp, $\beta$.precip, and $\beta$.int, describe the relationships between the all-species fruiting dates and temperature and precipitation, as well as their interaction. Errors, $\varepsilon_{s, y, z \prime}$ were assumed to come from a Normal distribution.

To test whether relationships with climate varied among different life-history groups, we allowed regression coefficients to vary according to life history:

$$
\begin{aligned}
\text { FruitDate }_{s, y, z}= & \alpha_{s, z}+\beta \cdot \text { temp }_{\text {life.history }} * \text { Temp }_{y, z} \\
& +\beta \cdot \text { precip }_{\text {life.history }} * \text { Precip }_{y, z}+\beta \cdot \text { int }_{\text {life history }} \\
& * \text { Precip }_{y, z} * \text { Temp }_{y, z}+\epsilon_{s, y, z}
\end{aligned}
$$

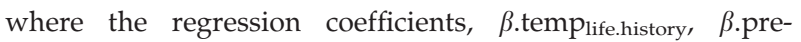
cip $_{\text {life.history, and } \beta \text {.int }}$ life.history, describe the relationships $_{\text {, }}$ between temperature, precipitation, and their interaction for each of four life-history groups: (i) mycorrhizal and associated with deciduous tree species; (ii) saprotrophic and associated with deciduous tree species; (iii) mycorrhizal and associated with evergreen tree species; and (iv) saprotrophic and associated with evergreen tree species. Coefficients were estimated independently for each life-history group.

To test whether species varied in their relationships with climate, we fit a similar model to the above, but with regression coefficients that varied among species:

$$
\begin{aligned}
\text { FruitDate }_{s, y, z}= & \alpha_{s, z}+\beta \cdot \text { temp }_{s} * \text { Temp }_{y, z}+\beta \cdot \text { precip }_{s} \\
& * \text { Precip }_{y, z}+\beta \cdot \text { int }_{s} * \operatorname{Precip}_{y, z} * \operatorname{Temp}_{y, z}+\epsilon_{s, y, z}
\end{aligned}
$$

where the regression coefficients, $\beta$.temp ${ }^{\prime} \beta$. precip $_{s}$, and $\beta$.int ${ }_{s}$ describe the relationships for each species, $s$, between temperature, precipitation, and their interaction, respectively. These species-level coefficients were estimated independently for each species.

Finally, as an additional test of how species' life-history characteristics affected their trends over time and responses to climate, while accounting for species-specific differences, we added to the above a species-level regression in which species-specific regression parameters $\left(\beta\right.$. temp $_{s}$ and $\beta$.precip pro $\left._{s}\right)$ were modeled as a function of life-history characteristics:

$$
\begin{aligned}
\beta \cdot \text { temp }_{\mathrm{s}}= & \alpha \cdot \text { temp }+\beta \cdot \text { life } \cdot \text { temp } \text { mycorStatus, HostPlant }+\beta \cdot \text { size } \\
& \cdot \text { temp } * \text { Size }_{\mathrm{s}}+\text { Genus } \cdot \text { temp }_{\mathrm{g}}+\epsilon_{\mathrm{s}},
\end{aligned}
$$

and

$$
\begin{aligned}
\beta \cdot \text { precip }_{\mathrm{s}}= & \alpha \cdot \operatorname{precip}+\beta \cdot \text { life } \cdot \text { precip }_{\text {mycorStatus, HostPlant }} \\
& +\beta \cdot \operatorname{size} \cdot \operatorname{precip} * \operatorname{Size}_{\mathrm{s}}+\text { Genus } \cdot \operatorname{precip}_{\mathrm{g}}+\epsilon_{\mathrm{s}},
\end{aligned}
$$

where $\beta$.life.temp and $\beta$.life.precip are fixed effects for each of the four combinations of mycorrhizal status (mycorrhizal or saprotrophic) and plant association (deciduous or coniferous associated). Size $_{s}$ is the maximum fruiting body size reported for each species, $s$, included to test the hypothesis that sensitivities to climate are related to mushroom size. Maximum size was calculated as the product of maximum cap diameter and stem length; two measures of size are often reported. Genus $_{g}$ is a genus-level random effect to account for nonindependence of species within genera. And $\alpha$.temp and $\alpha$.precip are overall intercepts for these species-level regressions. The resulting multilevel model allowed estimation of speciesspecific relationships to climate while also testing how species characteristics affected these relationships. Effects of life history on climate sensitivity were assessed by calculating contrasts between different $\beta$.life. For example, to test for differences in temperature sensitivity between mycorrhizal and saptrotrophic species associated with deciduous trees, the following contrast was calculated: $\beta$.life.temp $p_{\text {Mycorrhizal, }}$ Deciduous $-\beta$.life.temp $p_{\text {Saprotrophic, Deciduous. Comparable contrasts }}$ were calculated to test for differences among each of the four life-history groups. The difference between each life-history group was deemed statistically significant if the $95 \%$ credible 
interval of its contrast's posterior distribution did not overlap zero.

Trends over time. In addition to the uneven geographic distribution of herbarium data, the heterogeneous temporal distribution of collections further complicates efforts to estimate changes in fruiting date over time. To account for the uneven distribution of collections across years, we analyzed fruiting trends over decades instead of over years. To do this, we regressed the mean dates of fruiting within bins of 10 years for each species (from 1900 to 2000) vs. decade. Decadal means were estimated in the same model as the regression, thereby allowing uncertainty in means to be reflected in regression estimates. As a result, outliers driven by decades with few collections are less likely to influence regression estimates (see Data S1 for further illustration of this approach). Bins of 10 years were chosen as a balance between including enough data points within bins to be useful and having enough bins to estimate trends over time using regression. Therefore, we considered collection dates of each species, s, in any given year, $y$, and climate zone, $z$, to represent random draws from the true distribution of fruiting dates in that bin:

$$
\text { FruitDate }_{\mathrm{s}, \mathrm{y}, \mathrm{z}} \sim \operatorname{Normal}\left(\mu_{\mathrm{s}, \mathrm{d}, \mathrm{z}}, \sigma_{\mathrm{s}}^{2}\right)
$$

where $\mu_{s, d, z}$ is the mean fruiting date within decade, $d$, and $\sigma_{\mathrm{s}}^{2}$ is the estimated variance surrounding decadal means for species $s$. The regression of mean fruiting date vs. time was thus:

$$
\mu_{\mathrm{s}, \mathrm{d}, \mathrm{z}}=\alpha_{\mathrm{s}, \mathrm{z}}+\beta * D+\epsilon_{\mathrm{s}, \mathrm{d}}
$$

where $\alpha_{s, z}$ is an intercept term for each species and zone, $\beta$ is the regression coefficient describing the relationship between decade, D (1-14, spanning 1880-2010), and mean fruiting date. Errors, $\varepsilon_{s, d}$, were estimated for each species and decade (see Data S1 for additional detail). This results in higher uncertainty for decadal means with fewer data, thereby limiting the influence of poorly sampled decades.

To test whether species varied in their trends over time, we fit a similar model to the above, but with a regression coefficient that varied among species:

$$
\mu_{\mathrm{s}, \mathrm{d}, \mathrm{z}}=\alpha_{\mathrm{s}, \mathrm{z}}+\beta_{\mathrm{s}} * D+\epsilon_{\mathrm{s}, \mathrm{d}}
$$

where now the regression coefficients, $\beta_{s}$, describe the trend over time for each species, $s$.

All models were fit in a Bayesian framework, which was flexible to accommodate different hierarchical structures, unbalanced data, and facilitate using estimates for forecasting with uncertainty. We used noninformative priors for all intercepts, $\alpha$, regression coefficients, $\beta$, and errors, $\varepsilon$, as follows: $\alpha \sim$ Normal $(0,10000), \beta \sim$ Normal $(0,10000)$, and $\varepsilon$ were assigned uniform priors on the variances as $\sigma^{2} \sim$ Uniform (0, 10000). Models were fit and posterior densities of each parameter were obtained using OpenBUGS 1.4 (Lunn et al., 2009), called using the R2OpenBUGS package (Sturtz et al., 2005) of R ( R Development Core Team, 2008). Models were run for roughly 20,000 iterations and thinned by 10 to reduce autocorrelation of parameter samples. Convergence was assessed visually and by calculating the Gelman-Rubin statistic from three independent chains, and preconvergence 'burn-in' iterations were discarded.

\section{Results}

\section{Responses to climate}

When analyzed as an aggregate community, fungi fruited later in response to warmer summer temperatures and less summer precipitation (Fig. 1; partial regression coefficients with $95 \%$ credible intervals: $2.85(2.45,3.24)$ days per ${ }^{\circ} \mathrm{C}$ and $-0.53(-0.78,-0.28)$ days per $\mathrm{cm}$ precipitation). This aggregate model allowed mean fruiting dates to vary among species, but assumed consistent responses to climate. When responses to climate were allowed to vary among broad life-history groups, significant differences were found among trophic groups and tree associations (Fig. 1). Species associated with evergreen trees were significantly more delayed in warm years and more advanced in wet years compared with those associated with deciduous trees. The effect of mycorrhizal status depended on tree association. Among deciduous-associated species, saprotrophic species were more delayed than mycorrhizal species under higher temperatures and more advanced under higher precipitation.

When species were allowed to have individual responses to climate, they varied considerably in their responses to both temperature and precipitation (Figs 2 and 3). There were 29 species that fruited significantly later with higher temperatures, 21 species that fruited significantly earlier, and 224 species that had no significant responses to temperature (Fig. 2). Spring species, such as the Morchella genus (the morels), all tended to fruit earlier in warmer years. There were 23 species that fruited later in response to greater precipitation, 29 species fruiting earlier in response to greater precipitation, and 222 species with responses to precipitation that were not statistically significant (Fig. 3). Many species also had significant interaction terms, suggesting that the effects of temperature depended on precipitation (Fig. S2). Most of the significant interaction terms (53 of the 63 species with significant interactions) were negative, suggesting that increased temperature delayed phenology less under conditions of greater precipitation.

\section{Trends over time}

Analyzed as an aggregate community, there was a slight trend toward later fruiting over time of 0.18 days per decade (95\% credible interval: -0.07, 0.47; $P=0.078)$. Mycorrhizal species also exhibited a more 


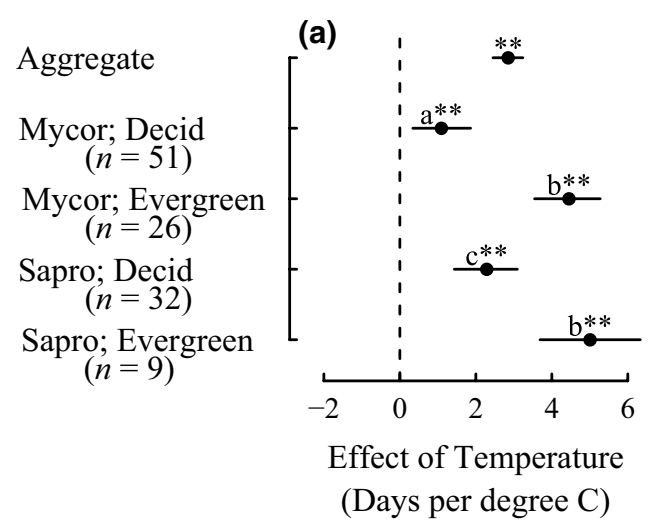

(d)

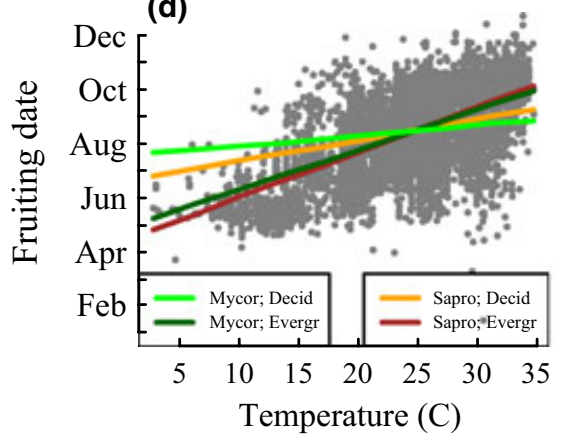

(b)

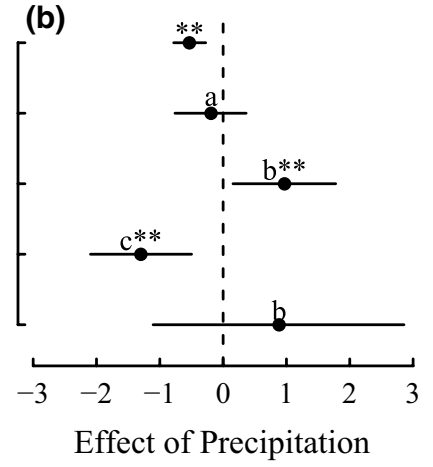

(Days $\mathrm{cm}^{-1}$ )

(e)

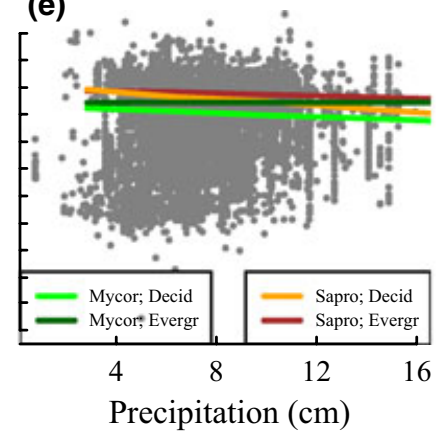

(c)

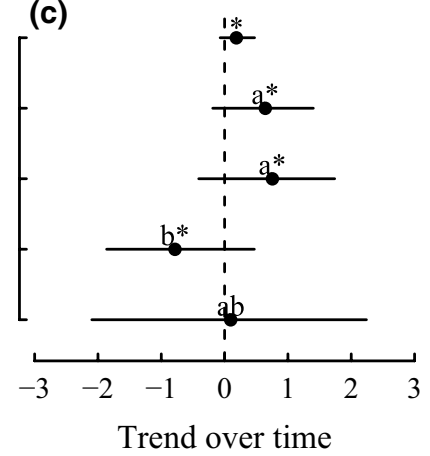

(Days per decade)

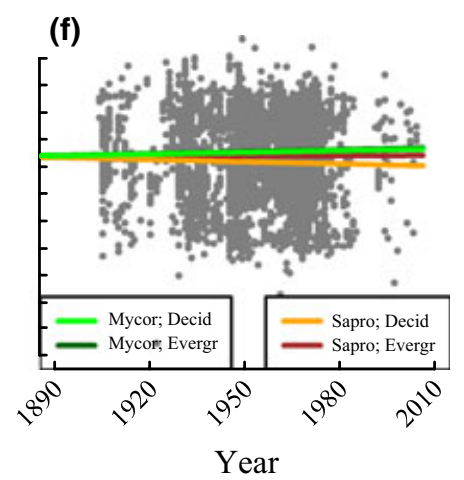

Fig. 1 Responses to climate and trends over time depend on life history. Regression coefficients (a-c) showed differences among lifehistory groups. Mean and $95 \%$ credible intervals are plotted. The 'aggregate' response at the top is from the simplest model that allows different intercepts for each species and climate zone, but assumes common responses. The intervals for each life-history group come from models that allow different slopes for each group. Letters signify statistical differences among groups, and asterisk show the differences in each response from zero $(* * P<0.05 ; * P<0.1)$. In bottom graphs $(\mathrm{d}-\mathrm{f})$ are plotted the raw fruiting data and predicted responses for each life-history group.

positive trend over time than saprotrophic species, particularly among deciduous-associated species (Fig. 1c). When allowing for variation in slopes among species, we found significant variation among species, with most species ( 267 of 274 species) not displaying a statistically significant trend (Fig. 4). Only six species showed a positive trend over time, and one showed a negative trend over time (Fig. 4). Trends over time were not related to sensitivities to climate (Fig. S3).

\section{Other relationships}

There was a positive relationship between sensitivity to temperature and the mean date of fruiting for a species, with later fruiting species exhibiting more delayed responses to temperatures $(P<0.01$; Fig. S4). However, mean date of fruiting was not related to sensitivity to precipitation nor trend over time (Fig. S4). Trends over time and sensitivities to climate were also not related to the number of collections (Fig. S5). Although species within some genera tended to respond similarly, there was significant variation among congeners (Fig. S6).

\section{Discussion}

Our results demonstrate that the timing of mushroom fruiting in Michigan is sensitive to climate, with the fungal community as a whole producing mushrooms later in years with higher temperatures and less precipitation. This pattern is consistent with the hypotheses that warm conditions encourage continued mycelial growth instead of fruiting and that increased precipitation accelerates fruiting. Greater seasonal precipitation may also be associated with earlier timing of rains, so both the quantity and timing of rainfall may be important and need to be included in forecasts of future phenology. Our results are consistent with a study from Norway that found later fruiting correlated with increased temperature in autumn and winter of the current and previous year (Kauserud et al., 2008). Our finding that spring species tended to fruit earlier under higher temperatures was also consistent with a study of spring species in Norway and United Kingdom in which earlier fruiting was associated with warmer winter temperatures (2010b). The overall mean sensitivity 

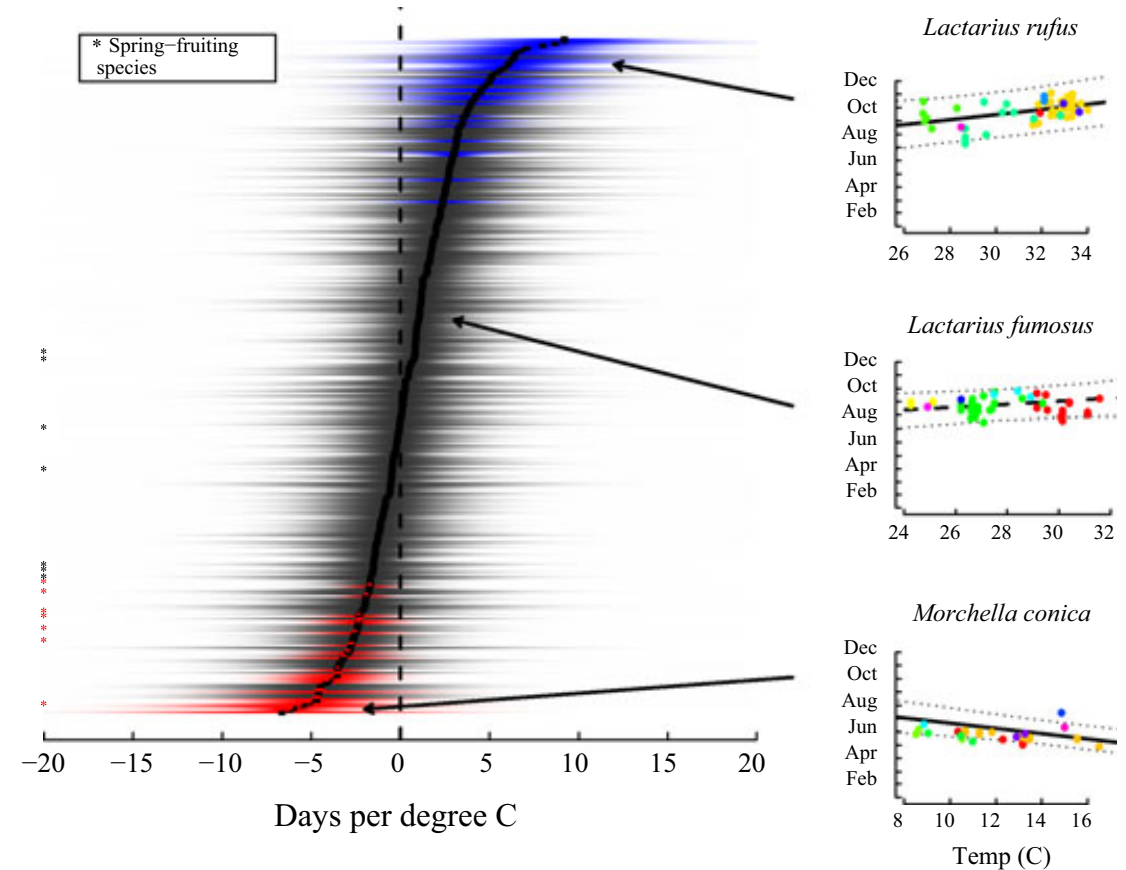

Fig. 2 Species-specific relationships between fruiting dates and mean summer temperature. Right graphs: On the right are plotted three example species' relationships between fruiting date and temperature. Points represent individual collections, and the colors are different climate station zones. The slopes of these relationships, i.e., the partial regression coefficients from the full model including precipitation, are plotted for each species on the left. Left graphs: Solid vertical bars represent the mean slopes of fruiting date vs. temperature, given mean precipitation values. Horizontal shaded bars represent the posterior probability distributions of these partial regression coefficients, so wider bars represent greater uncertainty in the relationship. Species with significantly later fruiting in response to higher temperature are colored blue (significance measured as $a>0.9$ probability that the relationship is greater than zero). Species with significantly earlier fruiting with higher temperatures are colored red (with $>0.9$ probability that the relationship is less than zero). Species plotted in gray showed no significant relationship with temperature. Spring-fruiting species are labeled with an asterisk $(*)$. Species names with parameter estimates can be found in supplementary material.

of these fungi to temperature is weaker than observed for plants. Spring plant phenology (leafing and flowering) has been found to be advancing at an average rate of 5-6 days per degree C (Wolkovich et al., 2012), compared to 2.85 days per degree $C$ found here. This comparison should be interpreted cautiously, however, due to the differences in data availability between fungi and plants.

The significant negative interactions between temperature and precipitation in our study also suggest a more complex set of responses to climate change than previously reported. In particular, the predominance of negative interactions suggests that the expected delays in phenology with climate warming may be lessened under conditions of greater precipitation, but enhanced when precipitation decreases. Because changes in rainfall patterns are expected to be regionally variable under climate change, this result suggests the need for regionally tailored predictions of fungal responses to climate change.

Our analyses also highlight two types of substantial variability in fungal phenology with important ecological and evolutionary consequences: variability among species in their responses to climate, and annual variability within species. The among-species variability was striking. Although many species fruited later in years with less precipitation and higher temperatures, there were a number of species with the opposite responses, and a majority of species that showed no significant responses. Although complicating efforts to forecast responses, this variation could increase the potential resilience of species networks to changing climate, as suggested for plants (Memmott et al., 2007; Kaiser-Bunbury et al., 2010) and may be compensated by changes in community structure.

Some of the differences in species' responsiveness to climate were related to basic life-history characteristics. We found that both trophic strategy (mycorrhizal vs. saprotrophic) and tree association (deciduous vs. coniferous) may help determine species' climate sensitivities (Fig. 1). In particular, fruiting of species associated with evergreen trees was more delayed in response to higher temperatures and less advanced with higher precipitation than deciduous-associated species (Fig. 1). An earlier study found deciduous-associated species to be more responsive over time than coniferous-associated 

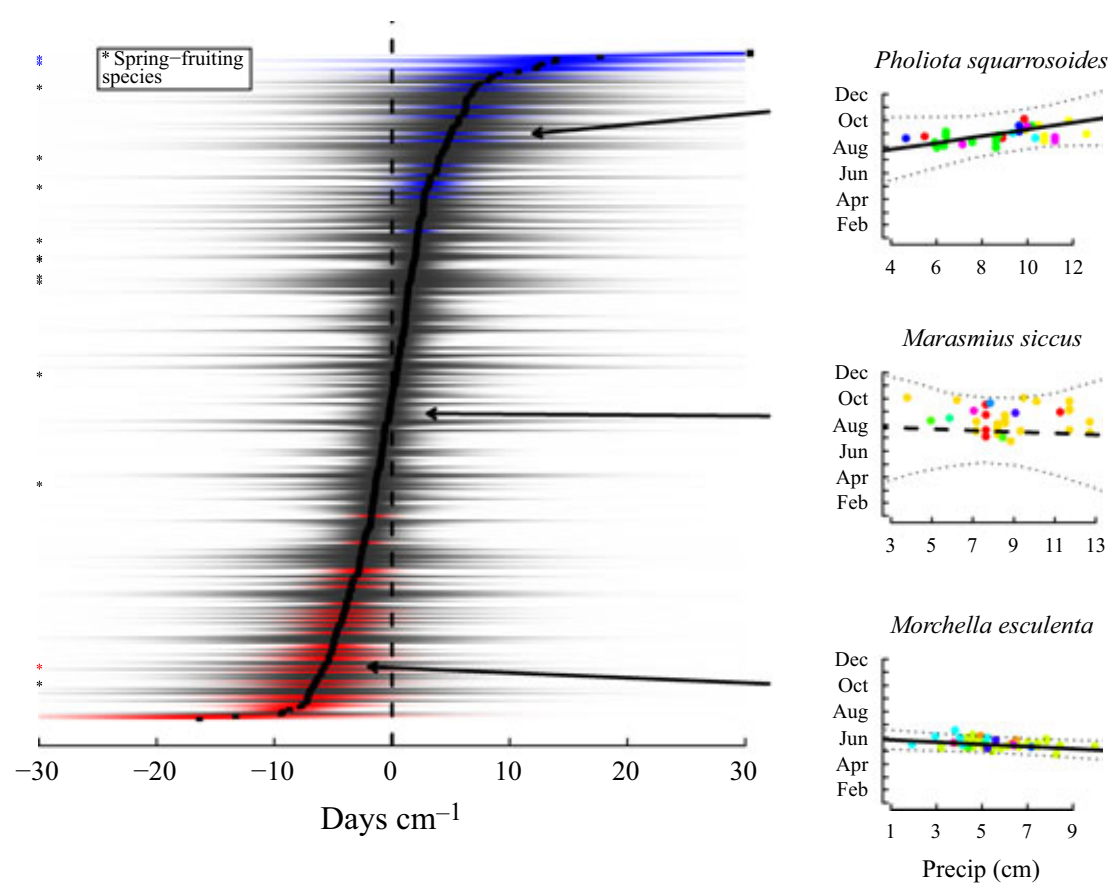

Fig. 3 Species-specific relationships between fruiting dates and mean summer precipitation. Right graphs: On the right are plotted three example species' relationships between fruiting date and precipitation. Points represent individual collections, and the colors are different climate station zones. The slopes of these relationships, i.e., the partial regression coefficients from the full model including temperature, are plotted for each species on the left. Left graphs: Solid vertical bars represent the mean slopes of fruiting date vs. precipitation, given mean temperature values. Horizontal shaded bars represent the posterior probability distributions of these partial regression coefficients, so wider bars represent greater uncertainty in the relationship. Species with significantly later fruiting in response to higher precipitation are colored blue (significance measured as $a>0.9$ probability that the relationship is greater than zero). Species with significantly earlier fruiting with higher precipitation are colored red (with $>0.9$ probability that the relationship is less than zero). Species plotted in gray showed no significant relationship with precipitation. Spring-fruiting species are labeled with an asterisk (*). Species names with parameter estimates can be found in supplementary material.

species (Gange et al., 2007), but responsiveness to temperature was not reported for these groups. Among deciduous-associated species, saprotrophic species showed a stronger delay in fruiting in response to higher temperatures and stronger advance with higher precipitation. Accompanying these differences in sensitivity to temperature and precipitation, deciduousassociated saprotrophic species also exhibited a trend toward early fruiting over time compared with other species (Fig. 1). These differences support the hypothesis that direct climate effects on fungi combine with indirect effects via host plants. Because the mycelium of ectomycorrhizal fungi is associated with tree roots, whereas saprotrophic species utilize the organic layer of soils, mycorrhizal species may be distributed deeper in the soil than saprotrophic species. As a result, ectomycorrhizal species may be expected to rely more on host trees for phenological cues than direct experience with temperature and precipitation. Because trees are largely extending their growing season in response to warmer summers (Piao et al., 2007), mycorrhizal fungi also exhibit this delay but may be constrained by tree phenology. In contrast, the more superficial location of saprotrophic species may mean they can more readily respond to increases in precipitation and extend their fruiting seasons in warmer years.

The high variability in fruiting dates within species, seen for example in the inset graphs in Figs 2-4, is common for fungi, and may result from a combination of genetic variability, phenotypic plasticity, and unexplained environmental or biotic cues for fruiting. To the degree that this variation is heritable, it may facilitate adaptation to changing conditions and buffer the overall effects of climate change on the species. Fruiting times have been shown to be related to basic life-history characteristics such as spore size (Kauserud et al., 2010a), but the broader evolutionary consequences remain largely unknown. The high variability also complicates the effort to quantify responses to ongoing climate change, particularly when using nonsystematic data sources such as herbaria. This may explain in part the numerous species with trends in their responses to climate, but with large uncertainty yielding statistically insignificant results. On the other hand, despite the 


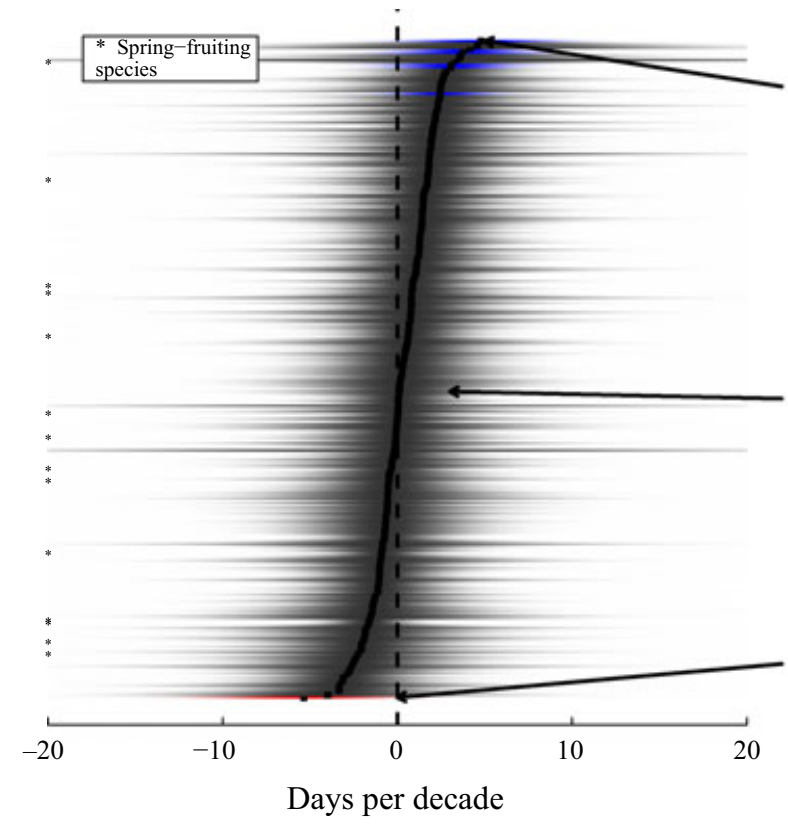

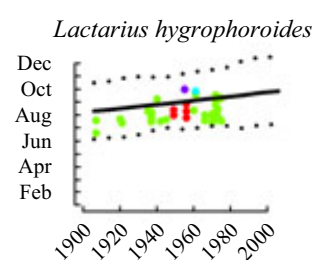
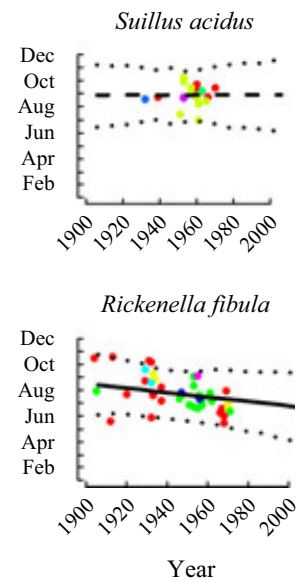

Fig. 4 Species-specific trends of fruiting dates over time. Right graphs: On the right are plotted three example species' relationships between fruiting date and year. Points represent individual collections and the colors are different climate station zones. The slopes of these relationships from the decadal model (described in methods) are plotted for each species on the left. Left graphs: Solid vertical bars represent the mean slopes of fruiting date over time. Horizontal shaded bars represent the posterior probability distributions of these partial regression coefficients, so wider bars represent greater uncertainty in the relationship. Species with significantly later fruiting over time are colored blue (significance measured as $a>0.9$ probability that the relationship is greater than zero). Species with significantly earlier fruiting over time are colored red (with $>0.9$ probability that the relationship is less than zero). Species plotted in gray showed no significant trend over time. Spring-fruiting species are labeled with an asterisk $(*)$. Species names with parameter estimates can be found in supplementary material.

challenges associated with herbarium data, it is striking that the patterns we have observed in North America mirror the same trends observed in Europe.

Some of the variability within species may also be explained by the presence of 'cryptic' species, nearly impossible to distinguish on the basis of morphology (Taylor et al., 2006). The presence of cryptic species in this study and other fungal phenology studies is likely to further reduce the ability to detect trends if cryptic species have different responses to climate. However, the differences among genera found in this study support the hypothesis that related species tend to respond similarly. We attempted to reduce systematic biases in species identification by limiting this study to confined geographic region and a single herbarium. However, because North America is comprised of such diverse ecoregions and climates, a holistic approach to understanding the effect of climate on fungal phenology will require integrating many more records across multiple herbaria.

The aggregate trend toward later fruiting over time (Fig. 1c) is consistent with previous studies of fall fungi (Kauserud et al., 2008). However, the rate of delay was small relative to other studies, and very few species exhibited significant trends in fruiting dates over time when analyzed separately (Fig. 3). There are several reasons for very cautious interpretation of trends over time in this and other studies. First, estimated trends over time are very sensitive to the time period of analysis (Diez et al., 2012), and most studies of historical data have uneven distributions of data through time. Here, the data in the UM herbarium are biased toward mid20th century, which could influence the lack of observed trends over time because the rate of climate change has generally increased toward the end of the 20th century. In contrast, relationships with climate are more robust because the annual climatic variability is much greater than mean trends over time. Nonetheless, climate stations used in this study showed only subtle, but significant, trends in temperature and precipitation over the past century (Fig. S7), which may also help to explain the paucity of significant phenological trends over time.

Another reason to cautiously interpret observed trends over time in all climate change studies is that other important factors besides climate have also been changing over time, risking false attribution to climate change. For example, fungal fruiting patterns may be 
influenced by ecosystem changes such as shifting forest composition (Shaw et al., 2003; Dickie et al., 2010), patterns of forestry and urbanization, nitrogen deposition (Gillet et al., 2010), and $\mathrm{CO}_{2}$ increases. Most of these additional drivers may indeed have changed over time and could affect fungal phenology. Although these factors may therefore complicate attribution of observed phenological trends over time to climate change, they do not bias the direct responses to temperature and precipitation measured in this study.

This study provides strong evidence for a link between climate and fungal phenology in North America that varies among species and is affected by life history. By focusing on direct linkages between climate and species responses, we suggest that predicted future climate warming will lead to later fruiting on average. This may lead to longer annual periods of vegetative growth and decomposition, thus increasing fungal respiration. This increased respiration under warmer conditions may contribute to offsetting the increase in carbon uptake by plant communities and belowground storage, thereby altering the net effects of climate change on the carbon cycle (Piao et al., 2008). This study further highlights how these responses to temperature will also depend on precipitation patterns, suggesting that future forecasting of ecosystem dynamics will need accurate predictions of both temperature and precipitation changes.

\section{Acknowledgements}

We are indebted to the mycologists who collected these specimens and deposited them in MICH. While data from 187 collectors were used here, the following 18 individuals accounted for ca. $93 \%$ of the data used here: A.H. Smith, V. Potter, F. Hoseney, J.F. Ammirati, H.D. Thiers, N.S. Weber, N.J. Smith, C.H. Kauffman, H.E. Bigelow, K.A. Harrison, R.L. Shaffer, M. Gilliam, C. Nimke, W. Patrick, I. Bartelli, E.B. Mains, J. LaRue, and S.J. Mazzer. We also thank the James lab group and Levine lab group for discussion of this project. Finally, we are very grateful to three anonymous reviewers for their time and comments on the manuscript.

\section{References}

Büntgen U, Kauserud H, Egli S (2012) Linking climate variability to mushroom productivity and phenology. Frontiers In Ecology And The Environment, 10, 14-19.

Büntgen U, Peter M, Kauserud H, Egli S (2013) Unraveling environmental drivers of a recent increase in Swiss fungi fruiting. Global Change Biology, 19, 27852794.

Clemmensen KE, Bahr A, Ovaskainen O et al. (2013) Roots and associated fungi drive long-term carbon sequestration in boreal forest. Science, 339, 1615-1618.

Dickie IA, Kaluck I, Stasin M, Oleksynd J (2010) Plant host drives fungal phenology. Fungal Ecology, 3, 311-315.

Diez J, Ibanez I, Inouye DW, Crimmins MA, Crimmins TM, Miller-Rushing AJ, Mazer SJ (2012) Forecasting phenology across scales: the challenge and value of variability. Ecology Letters, 15, 545-553.

Egli S (2011) Mycorrhizal mushroom diversity and productivity - An indicator of forest health? Annals of Forest Science, 68, 81-88.
Encinas-Viso F, Revilla TSA, Etienne RS (2012) Phenology drives mutualistic network structure and diversity. Ecology Letters, 15, 198-208.

Fogel R, Trappe JM (1978) Fungus Consumption (Mycophagy) by Small Animals. Northwest Science, 2, 1-31.

Gange A, Gange E, Sparks T, Boddy L (2007) Rapid and recent changes in fungal fruiting patterns. Science, 316, 71 .

Gillet F, Peter M, Ayer F, Bütler R, Egli S (2010) Long-term dynamics of aboveground fungal communities in a subalpine Norway spruce forest under elevated nitrogen input. In: Oecologia 164, 499-510.

Hegland SJ, Nielsen A, Lazaro A, Bjerknes AL, Totland O (2009) How does climate warming affect plant-pollinator interactions? Ecology Letters, 12, 184-195.

Kaiser-Bunbury CN, Muff S, Memmott J, Müller CB, Caflisch A (2010) The robustness of pollination networks to the loss of species and interactions: a quantitative approach incorporating pollinator behaviour. Ecology Letters, 13, 442-452.

Kauserud H, Stige LC, Vik JO, Okland RH, Hoiland K, Stenseth NC (2008) Mushroom fruiting and climate change. Proceedings Of The National Academy Of Sciences Of The United States Of America, 105, 3811-3814.

Kauserud H, Heegaard E, Halvorsen R, Boddy L, Holiland K, Stenseth NC (2010a) Mushroom's spore size and time of fruiting are strongly related: is moisture important? Biology Letters, 7, 273-276.

Kauserud H, Heegaard E, Semenov MA et al. (2010b) Climate change and spring-fruiting fungi. Proceedings Of The Royal Society B-Biological Sciences, 277, 1169-1177.

Kauserud H, Heegaard E, Buntgen U et al. (2012) Warming-induced shift in European mushroom fruiting phenology. Proceedings of the National Academy of Sciences, 109, 14488-14493.

Krebs C, Carrier P, Boutin S, Boonstra R, Hofer E (2008) Mushroom crops in relation to weather in the southwestern Yukon. Botany, 86, 1497-1502.

Kües U, Liu Y (2000) Fruiting body production in Basidiomycetes. Applied microbiology and biotechnology, 54, 141-152.

Lunn D, Spiegelhalter D, Thomas A, Best N (2009) The BUGS project: evolution, critique and future directions (with discussion). Statistics in Medicine, 28, 3049-3082.

Memmott J, Craze PG, Waser NM, Price MV (2007) Global warming and the disruption of plant-pollinator interactions. Ecology Letters, 10, 710-717.

Menzel A, Sparks T, Estrella N et al. (2006) European phenological response to climate change matches the warming pattern. Global Change Biology, 12, 1969-1976.

Moore D, Gange AC, Gange EG, Boddy L (2008) Fruit bodies: their production and development in relation to environment. In: Ecology of Saprotrophic Basidiomycetes (ed. Boddy L, Frankland JC, Van West P), pp. 79-102. Elsevier, London.

Orwin KH, Kirschbaum MUF, St John MG, Dickie IA (2011) Organic nutrient uptake by mycorrhizal fungi enhances ecosystem carbon storage: a model-based assessment. Ecology Letters, 14, 493-502.

Piao S, Friedlingstein P, Ciais P, Viovy N, Demarty J (2007) Growing season extension and its impact on terrestrial carbon cycle in the Northern Hemisphere over the past 2 decades. Global Biogeochemical Cycles, 21, 1-11.

Piao S, Ciais P, Friedlingstein P et al. (2008) Net carbon dioxide losses of northern ecosystems in response to autumn warming. Nature, 451, 49-52.

Pinna S, Gévry M-F, Côté M, Sirois L (2010) Factors influencing fructification phenology of edible mushrooms in a boreal mixed forest of Eastern Canada. Forest Ecology and Management, 260, 294-301.

R Development Core Team (2008) R: A Language and Environment for Statistical Computing. R Foundation for Statistical Computing, Vienna, Austria. ISBN 3-90005107-0. Available at: http:/ / www.R-project.org (accessed 10 January 2011).

Richardson A, Andy Black T, Ciais P et al. (2010) Influence of spring and autumn phenological transitions on forest ecosystem productivity. Philosophical Transactions of the Royal Society B: Biological Sciences, 365, 3227.

Root TL, Macmynowski DP, Mastrandrea MD, Schneider SH (2005) Human-modified temperatures induce species changes: Joint attribution. Proceedings Of The National Academy Of Sciences Of The United States Of America, 102, 7465-7469.

Shaw PJA, Kibby G, Mayes J (2003) Effects of thinning treatment on an ectomycorrhizal succession under Scots pine. Mycological Research, 107, 317-328.

Straatsma G, Ayer FO, Egli S (2001) Species richness, abundance, and phenology of fungal fruit bodies over 21 years in a Swiss forest plot. Mycological Research, 105, 515-523.

Sturtz S, Ligges U, Gelman A (2005) R2WinBUGS: A Package for Running WinBUGS from R. Journal of Statistical Software, 12, 1-16.

Taylor J, Turner E, Townsend J, Dettman J, Jacobson D (2006) (2006) Eukaryotic microbes, species recognition and the geographic limits of species: examples from the kingdom Fungi. Philosophical Transactions of the Royal Society B: Biological Sciences, 361, 1947-1963.

Wolkovich EM, Cook BI, Allen JA et al. (2012) Warming experiments underpredict plant phenological responses to climate change. Nature, 485, 494-497. 


\section{Supporting Information}

Additional Supporting Information may be found in the online version of this article:

Data S1. An expanded explanation of how trends over time were modeled given heterogeneous sampling.

Fig. S1. Study region. Map showing overall distribution of herbarium data across Michigan counties, along with location of climate stations (US Historical Climatology Network).

Fig. S2. Relationships between regression coefficients describing main effects of temperature (left) and precipitation (right) and interactions.

Fig. S3. Relationships between climate sensitivity and trends over time.

Fig. S4. Relationships between species' mean fruiting date and sensitivity to climate and trends over time.

Fig. S5. To test whether the amount of collecting effort affected estimated trends, we plotted relationships between the number of collections and estimated trends over time (a), responses to temperature (b), and responses to precipitation (c). Each point is a species' estimated response. None was significant.

Fig. S6. Responses of genera to climate. The genera analyzed in this study are listed along the $y$-axis with the number of species analyzed in parentheses. Gray circles are the mean responses of each species within the genus to temperature (a) and precipitation (b). Black circles and intervals show the means and \pm 1 SD for genera with multiple species.

Fig. S7. Climate trends in the region. Plotted are climate data for individual climate stations used in this study (gray lines) and the annual means of all stations (dark lines).

Table S1. A table of species analyzed in this study, along with their traits and estimated responses to climate. 